\title{
The Carbohydrate Threshold in Pregnancy and Gestational Diabetes: How Low Can We Go?
}

\author{
Arianne Sweeting 1,2,3 ${ }^{\mathbb{D}}$, Jovana Mijatovic ${ }^{2,4} \mathbb{D}$, Grant D. Brinkworth ${ }^{5,6}$, Tania P. Markovic ${ }^{1,2,7}$, Glynis P. Ross ${ }^{1,3}$, \\ Jennie Brand-Miller ${ }^{8}(\mathbb{D})$ and Teri L. Hernandez $9,10,11, * \mathbb{D}$
}

Citation: Sweeting, A.; Mijatovic, J.; Brinkworth, G.D.; Markovic, T.P.;

Ross, G.P.; Brand-Miller, J.;

Hernandez, T.L. The Carbohydrate Threshold in Pregnancy and Gestational Diabetes: How Low Can We Go? Nutrients 2021, 13, 2599.

https://doi.org/10.3390/nu13082599

Academic Editor: Clive J. Petry

Received: 6 June 2021

Accepted: 22 July 2021

Published: 28 July 2021

Publisher's Note: MDPI stays neutral with regard to jurisdictional claims in published maps and institutional affiliations.

Copyright: (c) 2021 by the authors. Licensee MDPI, Basel, Switzerland. This article is an open access article distributed under the terms and conditions of the Creative Commons Attribution (CC BY) license (https:// creativecommons.org/licenses/by/ $4.0 /)$.
1 Sydney Medical School, The University of Sydney, Sydney, NSW 2006, Australia; arianne.sweeting@sydney.edu.au (A.S.); tania.markovic@sydney.edu.au (T.P.M.); glynis.ross@health.nsw.gov.au (G.P.R.)

2 Charles Perkins Centre, Boden Initiative, University of Sydney, NSW 2006, Australia; jovana.mijatovic@ctc.usyd.edu.au

3 Department of Endocrinology, Royal Prince Alfred Hospital, Sydney, NSW 2050, Australia

4 NHMRC Clinical Trials Unit, University of Sydney, Sydney, NSW 2006, Australia

5 Commonwealth Scientific and Industrial Research Organisation-Health and Biosecurity, Sydney, NSW 2113, Australia; Grant.Brinkworth@csiro.au

6 School of Health Sciences, The University of Sydney, Sydney, NSW 2006, Australia

7 Metabolism \& Obesity Services, Royal Prince Alfred Hospital, Sydney, NSW 2050, Australia

8 School of Life and Environmental Sciences and Charles Perkins Centre, The University of Sydney, Sydney, NSW 2006, Australia; jennie.brandmiller@sydney.edu.au

9 College of Nursing, Anschutz Medical Campus, University of Colorado, Aurora, CO 80045, USA

10 Department of Medicine, Division of Endocrinology, Metabolism, and Diabetes, Anschutz Medical Campus, University of Colorado, Aurora, CO 80045, USA

11 Children's Hospital Colorado, Aurora, CO 80045, USA

* Correspondence: teri.hernandez@cuanschutz.edu; Tel.: +1-303-724-8538

Abstract: The original nutrition approach for the treatment of gestational diabetes mellitus (GDM) was to reduce total carbohydrate intake to $33-40 \%$ of total energy (EI) to decrease fetal overgrowth. Conversely, accumulating evidence suggests that higher carbohydrate intakes $(60-70 \%$ EI, higher quality carbohydrates with low glycemic index/low added sugars) can control maternal glycemia. The Institute of Medicine (IOM) recommends $\geq 175 \mathrm{~g} / \mathrm{d}$ of carbohydrate intake during pregnancy; however, many women are consuming lower carbohydrate (LC) diets $(<175 \mathrm{~g} / \mathrm{d}$ of carbohydrate or $<40 \%$ of EI) within pregnancy and the periconceptual period aiming to improve glycemic control and pregnancy outcomes. This report systematically evaluates recent data (2018-2020) to identify the LC threshold in pregnancy in relation to safety considerations. Evidence from 11 reports suggests an optimal carbohydrate range of 47-70\% EI supports normal fetal growth; higher than the conventionally recognized LC threshold. However, inadequate total maternal EI, which independently slows fetal growth was a frequent confounder across studies. Effects of a carbohydrate intake $<175 \mathrm{~g} / \mathrm{d}$ on maternal ketonemia and plasma triglyceride/free fatty acid concentrations remain unclear. A recent randomized controlled trial (RCT) suggests a higher risk for micronutrient deficiency with carbohydrate intake $\leq 165 \mathrm{~g} / \mathrm{d}$ in GDM. Well-controlled prospective RCTs comparing LC $(<165 \mathrm{~g} / \mathrm{d})$ and higher carbohydrate energy-balanced diets in pregnant women are clearly overdue.

Keywords: pregnancy; low carbohydrate; birth weight; micronutrients; ketones; lipids

\section{Introduction}

Nutrition therapy remains the foundation of GDM treatment. A recent meta-analysis [1] showed that enhancing nutritional quality after GDM diagnosis, irrespective of the specific dietary approach, improved maternal fasting and postprandial glycemia, and reduced excessive birthweight (BW). However, to date no nutritional strategy in GDM has completely normalized maternal and fetal outcomes [2,3]. The original nutritional approach, 
which lowered total carbohydrate intake to $33-40 \%$ of total energy (EI) [4], reduced postprandial glycemia and fetal overgrowth patterns (defined as macrosomia [BW $>4000 \mathrm{~g}$ ], large-for-gestational-age [LGA] or increased adiposity). Conversely, a separate body of evidence suggests higher carbohydrate intakes $(60-70 \%$ EI) incorporating high quality carbohydrate sources of lower glycemic index and added sugars can also control maternal glycemia [5-8]. This suggests a range of nutritional patterns can be effective in GDM management, enabling personalized interventions [2] that optimize adherence and reduce the need for adjunctive medication.

Currently, the IOM recommends at least $175 \mathrm{~g} / \mathrm{d}$ of carbohydrate intake during pregnancy [9]. However, many women are consuming lower carbohydrate (LC) diets during pregnancy and the periconceptual period [10-13] ( $<175 \mathrm{~g} / \mathrm{d}$ [9] or $<40 \%$ of EI [14]) in an attempt to improve glycemic control and pregnancy outcomes, but it remains unclear if this dietary practice is safe and appropriate to support maternal metabolic needs and optimal fetal growth. Safety concerns of LC diets in pregnancy relate to four key factors: (1) maintenance of the maternal/fetal glucose concentration gradient, relating to fetal growth and brain development $[9,15,16]$; (2) increased fetal exposure to maternal ketones, linked to impaired fetal and postnatal neural development [17-21]; (3) micronutrient deficiency risk [22,23]; and (4) risk of fetal exposure to increased maternal triglycerides (TG) or free fatty acids (FFA), linked to overgrowth [24-28]. In our view, there is a critical need to establish an acceptable LC intake threshold in pregnancy and GDM that safely supports maternal and fetal metabolic needs. This report systematically evaluates recent available data (2018-2020) to identify a LC threshold in pregnancy in relation to safety considerations.

\section{Materials and Methods}

To accelerate translation of newly generated knowledge into clinical practice, this report evaluates findings from the most recently published clinical trials and cohort studies [29]. Four specific questions based on safety concerns related to LC diets in pregnancy (listed below), guided our literature search. Reports published between 2015-2020 were initially identified in PubMed using keyword combinations including: "low carbohydrate", "pregnancy", "fetal growth", "high fat diet", "fetal growth", "diet pattern", "birth weight (BW)", "fetus", "maternal ketones", "betahydroxybutyrate", "gestational diabetes", "carbohydrate intake", "micronutrients". To be included, reports were then limited to those published between 2018-2020 [29]. Reports were published in English, were human studies in GDM or normal pregnancy, and represented original randomized controlled trials (RCTs) or oTablebservational investigations. Review papers and systematic reviews were excluded. A reported validated measure of dietary carbohydrate intake was necessary to evaluate if intake was related to fetal growth, ketone exposure, micronutrient deficiency, or increased maternal TG or FFA.

A total of 1398 abstracts and/or full text manuscripts were screened. After elimination of duplicates, and studies that did not meet inclusion criteria, 11 reports met inclusion criteria (Tables 1-4). Of these, 9 reported data from observational cohort or cross-sectional studies [30-38], 1 from an original RCT [39], and 1 from a secondary analysis of an RCT [40]. Figure 1 shows the distribution of carbohydrate intakes reported across these studies. Results are presented in alignment with the safety questions that guided the literature search. Historical context for each safety consideration is provided as further background. 


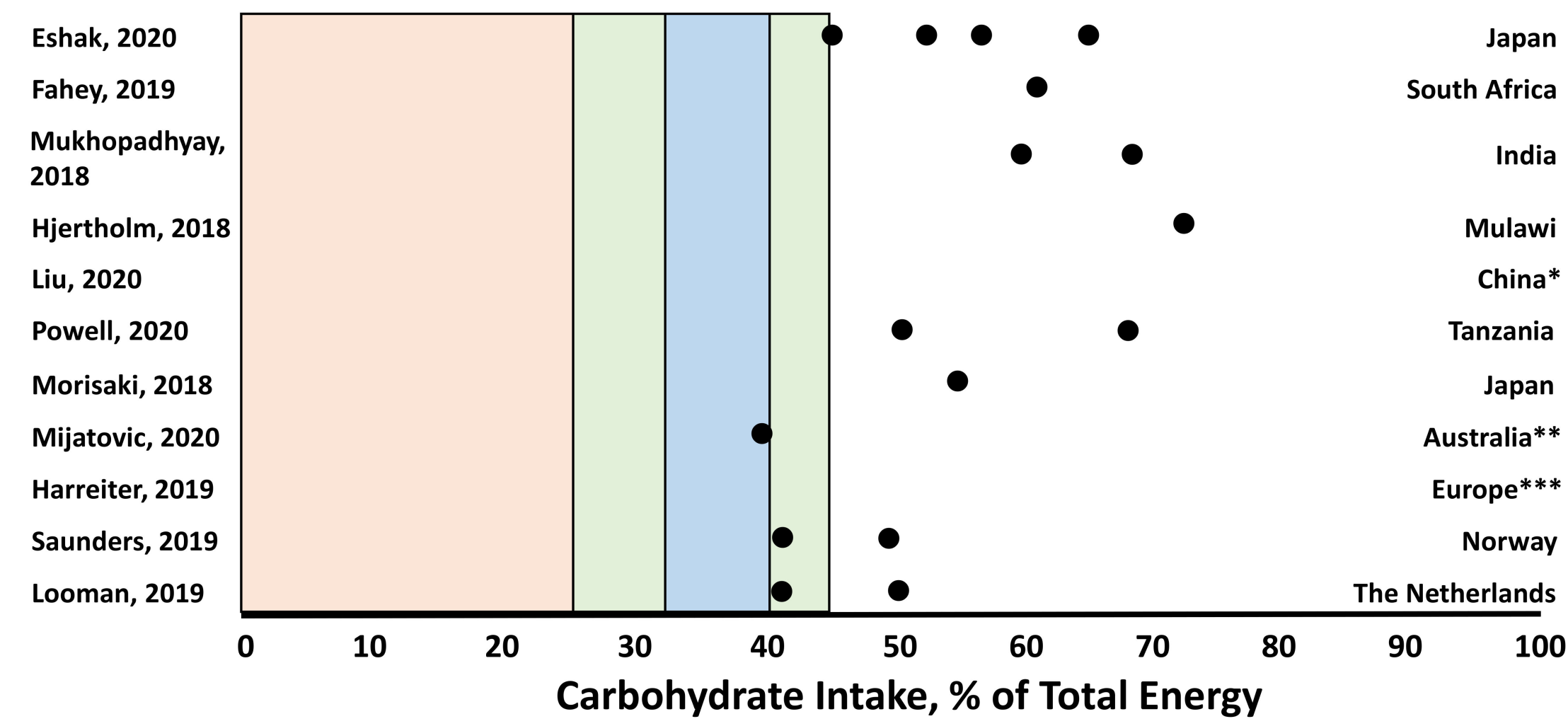

$\square$ Low-carbohydrate intake, pregnancy: $33-40 \%$

$\square$ Low-carbohydrate intake, non-pregnant: $26-45 \%$

*Diet patterns reported. Unable to estimate \%EI from carbohydrate

**Both groups, $38-39 \%$ of Energy

***Portions (not actual intake) reported

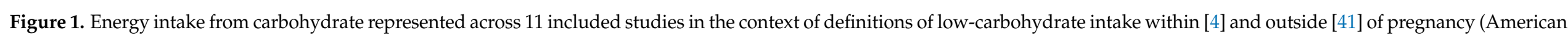
Diabetes Association consensus statement). Unless reported in Tables 1-4, energy from carbohydrate was calculated based on $4 \mathrm{kcal} / \mathrm{g}$ as a percentage of total EI. $(1 \mathrm{kcal}=4.184 \mathrm{~kJ})$. 


\section{Results \& Discussion}

\subsection{Question 1: Does a LC Diet in Pregnancy Compromise the Maternal-Fetal Glucose Concentration Gradient?}

Background. The human fetus relies on glucose for $\sim 80 \%$ of its energy requirements with maternal glucose as the dominant substrate that supports growth and brain development [15]. Glucose moves across the placenta via facilitated diffusion, dependent on a higher maternal concentration relative to the fetus [42]. The IOM guideline for carbohydrate intake of $\geq 175 \mathrm{~g} / \mathrm{d}$ is based on the estimated average requirement (EAR; to meet the needs of $\sim 50 \%$ of a population) for carbohydrate outside of pregnancy $(100 \mathrm{~g} / \mathrm{d})$, with an additional $33 \mathrm{~g}$ to support fetal brain development. When calculated as the recommended daily allowance (RDA; to meet the needs of $97-98 \%$ of a population), the following equation establishes the carbohydrate intake recommendation in pregnancy: $(100 \mathrm{~g} / \mathrm{d}$ [EAR outside of pregnancy] $+33 \mathrm{~g}$ [fetal brain development; rounded to $35 \mathrm{~g}$ ]) $+2 \times$ population coefficient of variation at $15 \%=175 \mathrm{~g} / \mathrm{d}$ [9]. Recent evidence suggests the human placenta consumes more glucose than originally assumed [16], a factor not considered within the IOM recommendation. Theoretically, if the maternal diet is too low in carbohydrate and maternal glucose levels remain low, the plasma maternal-fetal glucose gradient may be compromised, jeopardizing fetal growth. Models of maternal undernutrition have demonstrated that inadequate total maternal EI is tightly associated with reduced and stunted fetal growth patterns [43], but it is often not possible to separate the role of insufficient energy vs. carbohydrate intake. Moreover, both restricted and very high protein intake has been associated with low birth weight [44-46].

Review. Several studies suggest that birth size varies by carbohydrate intake. In a Japanese cohort of 78,793 (Table 1) [30], women were categorized into quartiles of carbohydrate intake (45.1\% to $64.9 \%$ of EI) and total EI. BW was lowest in the lowest quartile for both dietary factors. Birth length increased and ponderal index decreased with increasing carbohydrate intake. Similarly, the highest incidence of low BW $(<2500 \mathrm{~g})$ was observed in the lowest EI quartile based on a prior analysis of an expanded cohort $(n=91,637)[36]$ that showed neonates of women who consumed $<47 \%$ of EI from carbohydrate had the lowest BWs, independent of total EI (Table 1). Importantly, higher fat intake $>35 \%$ of EI, which often parallels LC intake, was also associated with lower BW. In South Africa, where carbohydrate intake follows a cyclical pattern with rainfall, harvest, gardening, and lean seasons [31], birth size z-scores increased with higher levels of carbohydrate consumption. However, whether total EI also fluctuated seasonally was unclear. Among pastoralist pregnant women in Tanzania [35], reduced EI during late pregnancy is a cultural tradition observed to prevent delivery complications related to larger BW. Women were found to reduce their EI by nearly $50 \%$ and carbohydrate intake by $64 \%$ to $\sim 100 \mathrm{~g} / \mathrm{d}$. Compared to infants born in urban Tanzania where EI remained constant, BWs $<2500 \mathrm{~g}$ were higher ( $31 \%$ vs. $12 \%)$. Interestingly, brain growth assessed by head circumference $(\mathrm{HC})$ in relation to total weight was compromised. BWs were lower but HCs were even lower $(1.7 \mathrm{SD})$ than the WHO standard, and $40 \%(n=46)$ were microcephalic (vs. $n=2$ infants in urban comparison group). Similarly, an RCT comparing a LC intake (165 g/d) vs. routine care (190 g/d) in GDM (Table 1) [39], showed no differences in BW, body composition, small-for-gestational-age (SGA) or LGA incidence. However, neonates of women in the LC group had smaller HCs (adjusted for weight gain, gestational age, and infant sex), that could also have been related to lower total EI in the LC group. In contrast, a multi-site RCT secondary analysis [40] of a Healthy Eating (HE) lifestyle modification starting at $<20$ weeks' gestation in women with obesity showed a reduced carbohydrate portion intake (g/d, \%EI not reported) did not result in differences in BW, LGA, or SGA. Overall, only the data from Japan [36] suggest a LC threshold of $<45-47 \%$ independent of total EI was associated with reduced fetal growth. Importantly, across studies (Figure 1) LC intakes tended to be accompanied by lower total EIs, a confounding factor that must be controlled to evaluate the independent effects of LC intake on fetal growth. No study reported maternal glucose concentrations. 


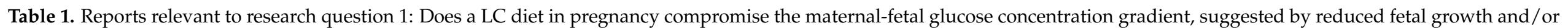
size at birth?

\begin{tabular}{|c|c|c|c|c|}
\hline Report & $\begin{array}{l}\text { Study Design } \\
\text { Population }\end{array}$ & $\begin{array}{l}\text { Carbohydrate Intake: } \\
\text { Measurement and Amount }\end{array}$ & Statistical Adjustment & $\begin{array}{c}\text { Carbohydrate Relationship to } \\
\text { Perinatal Concern }\end{array}$ \\
\hline Eshak, 2020 [30] & $\begin{array}{l}\text { Observational birth cohort } \\
\qquad n=78,793 \\
\text { Healthy pregnant women }(39.6 \% \\
\text { primiparous, } 31 \pm 5 \text { years }) \\
78 \% \text { had BMI } 18.5 \text { to }<25 \mathrm{~kg} / \mathrm{m}^{2} \\
\text { Mean gestational age at delivery } \\
38.9 \pm 1.5 \text { weeks } \\
\text {-Mean birth weight reported in } \\
\text { right column } \\
\text { Japan (15 regions represented) }\end{array}$ & $\begin{array}{c}\text { Food frequency questionnaire } \\
\text { Trimester } 2 \\
\\
\text { Median (IQR) CHO intake } 223.8 \\
(182.6-272.4) \mathrm{g} / \mathrm{d} \\
55.3 \% \text { of total energy intake } \\
\text { 61.9\% of women consumed } \\
<\text { recommended amount of } \mathrm{CHO} / \mathrm{d} \\
(57.5 \%) \\
\\
\text { Quartiles of } \mathrm{CHO} \% \text { Intake } \\
\text { Q1: } 45.1 \%(1075 \mathrm{kcal} / \mathrm{d}) \\
\text { Q2: } 52.9 \%(1466 \mathrm{kcal} / \mathrm{d}) \\
\text { Q3: } 57.7 \%(1800 \mathrm{kcal} / \mathrm{d}) \\
\text { Q4: } 64.9 \%(2650 \mathrm{kcal} / \mathrm{d})\end{array}$ & $\begin{array}{c}\text { Geometric means of nutrients } \\
\text { adjusted for: } \\
\text { Maternal age } \\
\text { Height } \\
\text { Education } \\
\text { Household income } \\
\text { Pre-pregnancy BMI } \\
\text { Net weight change in pregnancy } \\
\text { Smoking } \\
\text { Alcohol } \\
\text { Thyroid disease } \\
\text { Use of folate supplement } \\
\text { Offspring sex, gestational age at } \\
\text { delivery }\end{array}$ & $\begin{array}{c}\text { CHO and total energy intake were associated } \\
\text { with fetal growth (fully adjusted models) } \\
\text { Q1-3 CHO\%: Increased birth weight by quartile } \\
3030 \mathrm{~g} \rightarrow 3031 \mathrm{~g} \rightarrow 3037 \mathrm{~g} \rightarrow 3030 \mathrm{~g}(p=0.07) \\
\text { Q1-Q4 CHO } \% \text { : Increased birth length and } \\
\text { decreased ponderal index by quartile ( } p=0.002, \\
\quad p=0.02 \text {, respectively) } \\
\text { Q1-Q4 kcal/d: Increased birth weight by quartile } \\
3026 \mathrm{~g} \rightarrow 3031 \mathrm{~g} \rightarrow 3036 \mathrm{~g} \rightarrow 3036 \mathrm{~g}(p=0.004) \\
* 83.9 \% \text { of women consumed }<2500 \mathrm{kcal} / \mathrm{d} \\
\quad \text { (recommended amount) } \\
\text { Birth weight }<2500 \mathrm{~g} \text { by energy intake quartile } \\
8.4 \% \rightarrow 7.6 \% \rightarrow 7.2 \% \rightarrow 7.1 \%(p<0.001) \\
\text {-Fat intake was inversely associated with } \\
\text { ponderal index }(p=0.05) \\
\text {-Protein intake was not associated with } \\
\text { fetal growth }\end{array}$ \\
\hline
\end{tabular}


Table 1. Cont.

\begin{tabular}{|c|c|c|c|c|}
\hline Report & $\begin{array}{l}\text { Study Design } \\
\text { Population }\end{array}$ & $\begin{array}{c}\text { Carbohydrate Intake: } \\
\text { Measurement and Amount }\end{array}$ & Statistical Adjustment & $\begin{array}{c}\text { Carbohydrate Relationship to } \\
\text { Perinatal Concern }\end{array}$ \\
\hline Fahey, 2019 [31] & $\begin{array}{c}\text { Observational birth cohort } \\
n=752 \text { mother } / \text { infant dyads } \\
\text { Pregnant women ( } 43 \% \text { primiparous, } \\
14 \% \text { HIV positive, } \\
26.4 \pm 6.3 \text { years) } \\
\text { Mean gestational age at delivery } \\
39.3 \pm 2.3 \text { weeks } \\
\text { Mean birth weight } 3125 \pm 452 \mathrm{~g} \\
\text { Vehmbe District, Limpopo Provence, } \\
\text { South Africa }\end{array}$ & $\begin{array}{c}\text { Food frequency questionnaire at } \\
\text { delivery, to account for intake } \\
1 \text { month before delivery: } \\
61 \pm 10 \% \text { CHO } \\
24 \pm 8.2 \% \text { Fat } \\
13 \pm 2.9 \% \text { Protein } \\
\\
\text { Rainfall: November-February } \\
\text { Harvest: March-June } \\
\text { Gardening: July-October } \\
\text { Lean: November-February, } \uparrow \text { food } \\
\text { insecurity }\end{array}$ & $\begin{array}{c}\text { Models of dietary intake adjusted } \\
\text { for: } \\
\text { Maternal parity } \\
\text { HIV status } \\
\text { Height } \\
\text { Education } \\
\text { Marital status } \\
\text { Household income } \\
\text { Duration of pregnancy } \\
\text { Models of birth size z-score adjusted } \\
\text { for: } \\
\text { Maternal parity } \\
\text { HIV status } \\
\text { Height } \\
\text { Education } \\
\text { Marital status } \\
\text { Household income }\end{array}$ & $\begin{array}{c}\mathrm{CHO} \text { intake highest in lean season (64\%, January) } \\
\text { and lowest at end of Harvest ( } 56 \% \text {, June) } \\
\text { Fat intake was lowest in lean ( } 21 \% \text {, January) and } \\
\text { highest in Harvest season ( } 28 \% \text {, June) } \\
\text { Birth size z-scores (weight, length, head } \\
\text { circumference) peaked at lean season onset } \\
\text { (November), declined, and were lowest at } \\
\text { gardening season onset. } \\
\text { Birth size scores tracked with seasonal CHO } \\
\text { intake, where higher CHO intake was associated } \\
\text { with higher birth size scores and vice versa }\end{array}$ \\
\hline
\end{tabular}


Table 1. Cont.

\begin{tabular}{|c|c|c|c|c|}
\hline Report & $\begin{array}{l}\text { Study Design } \\
\text { Population }\end{array}$ & $\begin{array}{c}\text { Carbohydrate Intake: } \\
\text { Measurement and Amount }\end{array}$ & Statistical Adjustment & $\begin{array}{c}\text { Carbohydrate Relationship to } \\
\text { Perinatal Concern }\end{array}$ \\
\hline $\begin{array}{c}\text { Mukhopadhyay, } 2018 \\
\text { [32] }\end{array}$ & $\begin{array}{c}\text { Observational birth cohort } \\
n=1837 \\
\text { Healthy pregnant women (59\% } \\
\text { primiparous, } 24.4 \pm 3.8 \text { years, BMI } \\
\left.\sim 22 \pm 4 \mathrm{~kg} / \mathrm{m}^{2}\right) \\
\text { Gestational age at delivery } \\
38.6 \pm 1.5 \text { weeks } \\
\text { Mean birth weight } 2875 \pm 450 \mathrm{~g} \\
\text { ( } 28 \% \text { SGA rate) } \\
\text {-Women with an SGA infant were } \\
\text { younger ( } 0.5 \text { year), shorter }(0.1 \mathrm{~m}), \\
\text { weighed } \sim 3 \mathrm{~kg} \text { less and were more } \\
\text { often primiparous) } \\
\text { Bangalore, India }\end{array}$ & $\begin{array}{c}\text { Food frequency questionnaire } \\
\text { Trimester } 1 \\
\text { Total energy: } 1910 \pm 517 \mathrm{kcal} \\
\text { CHO: } 64.6 \pm 5.1 \% \\
\text { Fat: } 23.9 \pm 4.4 \% \\
\text { Protein: } 11.5 \pm 1.1 \% \\
\text { No differences in macronutrient } \\
\text { intake between those with AGA vs. } \\
\text { SGA infant } \\
\text { Categories for low, adequate, high } \\
\text { macronutrients: } \\
\text { CHO: low }<60 \%, \text { high }>70 \% \\
\text { Fat: low }<20 \%, \text { high }>25 \% \\
\text { Protein: low }<10 \%, \text { high }>20 \%\end{array}$ & $\begin{array}{c}\text { Macronutrient intakes adjusted for } \\
\text { total energy intake (nutrient } \\
\text { density method) } \\
\text { AORs accounted for: } \\
\text { Maternal age } \\
\text { Education } \\
\text { Parity } \\
\text { Height } \\
\text { Weight at recruitment } \\
\text { Fetal sex } \\
\text { Total energy intake }\end{array}$ & $\begin{array}{c}\text { Male births only: } \\
\text { Risk of SGA was higher with higher CHO intake } \\
\text { (aOR per 5\% energy: } 1.15 \text { [1.01-1.32]) } \\
\text { Risk of SGA was lower with lower fat intake } \\
\text { (aOR per 5\% energy: } 0.83 \text { [0.71-0.97]) } \\
\text { Categorical analysis } \\
\text { In women with high CHO intake }(\geq 334 \mathrm{~g} / \mathrm{d}) \text { : } \\
29 \% \text { SGA rate } \\
\text { aOR for SGA: } 1.67 \text { [1.002-2.780], } p=0.049 \\
\text { In women with high fat intake: } \\
26 \% \text { SGA rate } \\
\text { aOR for SGA: } 0.61 \text { [0.41-0.90], } p=0.01 \\
\text {-This was only true for male infants }\end{array}$ \\
\hline
\end{tabular}


Table 1. Cont.

\begin{tabular}{|c|c|c|c|c|}
\hline Report & $\begin{array}{l}\text { Study Design } \\
\text { Population }\end{array}$ & $\begin{array}{l}\text { Carbohydrate Intake: } \\
\text { Measurement and Amount }\end{array}$ & Statistical Adjustment & $\begin{array}{c}\text { Carbohydrate Relationship to } \\
\text { Perinatal Concern }\end{array}$ \\
\hline Hjertholm, 2018 [33] & $\begin{array}{l}\text { Cross-sectional with random } \\
\text { sampling } \\
n=132 \\
\text { Maternal characteristics not } \\
\text { reported } \\
\text { Mean birth weight } 3104 \pm 401 \mathrm{~g} \\
\text { (6\% had 'low birth weight') } \\
\text { Nankumba Traditional Authority, } \\
\text { Mangochi District, Malawi }\end{array}$ & $\begin{array}{l}\text { Over a } 10 \mathrm{~d} \text { period: } 3 \text {-d repeated } \\
\text { interactive multi-pass } 24-\mathrm{h} \text { recall } \\
\text { Collected during post-harvest } \\
\text { season, } 28 \text {-35 weeks' gestation } \\
\text { (mean week of collection } \\
\text { not reported) } \\
\text { Median (IQR) intake: } \\
\text { Energy: } 2096.5 \mathrm{kcal}(1778.1,2570.6) \\
\text { CHO: } 377 \mathrm{~g}(306,454), \sim 72 \% \\
\text { Fat: } 37.5 \mathrm{~g}(21.9,51.7), \sim 16 \% \\
\text { Protein: } 55 \mathrm{~g}(46,67), \sim 10 \% \\
\sim 1 \% \text { of women consumed }<135 \mathrm{~g} \\
\text { CHO/d and } 60.6 \% \text { consumed }<59 \mathrm{~g} \\
\text { protein/d (both the estimated } \\
\text { average requirement for pregnancy) }\end{array}$ & $\begin{array}{c}\text { Associations adjusted for: } \\
\text { Maternal age } \\
\text { Weight } \\
\text { Height } \\
\text { Gestational age } \\
\text { Literacy } \\
\text { Marital status } \\
\text { Household assets } \\
\text { Parity } \\
\text { Maternal energy intake } \\
\text { Newborn gender }\end{array}$ & $\begin{array}{l}\text { With each } 1 \% \uparrow \text { in fat intake, there was a } 0.1 \mathrm{~cm} \\
\text { increase in birth length and abdominal } \\
\text { circumference } \\
\text { With each } 1 \% \uparrow \text { in } \mathrm{CHO} \text { intake, there was a } \\
0.1 \mathrm{~cm} \text { decrease in birth length and abdominal } \\
\text { circumference } \\
\text { CHO intake was negatively associated with head } \\
\text { circumference ( } \beta \leq-0.01, p=0.04 \text { ) [small effect] } \\
\text { Adjusted for energy intake } \\
\text {-Most CHO intake in this region is accounted for } \\
\text { by nisma (porridge made from maize) }\end{array}$ \\
\hline
\end{tabular}


Table 1. Cont.

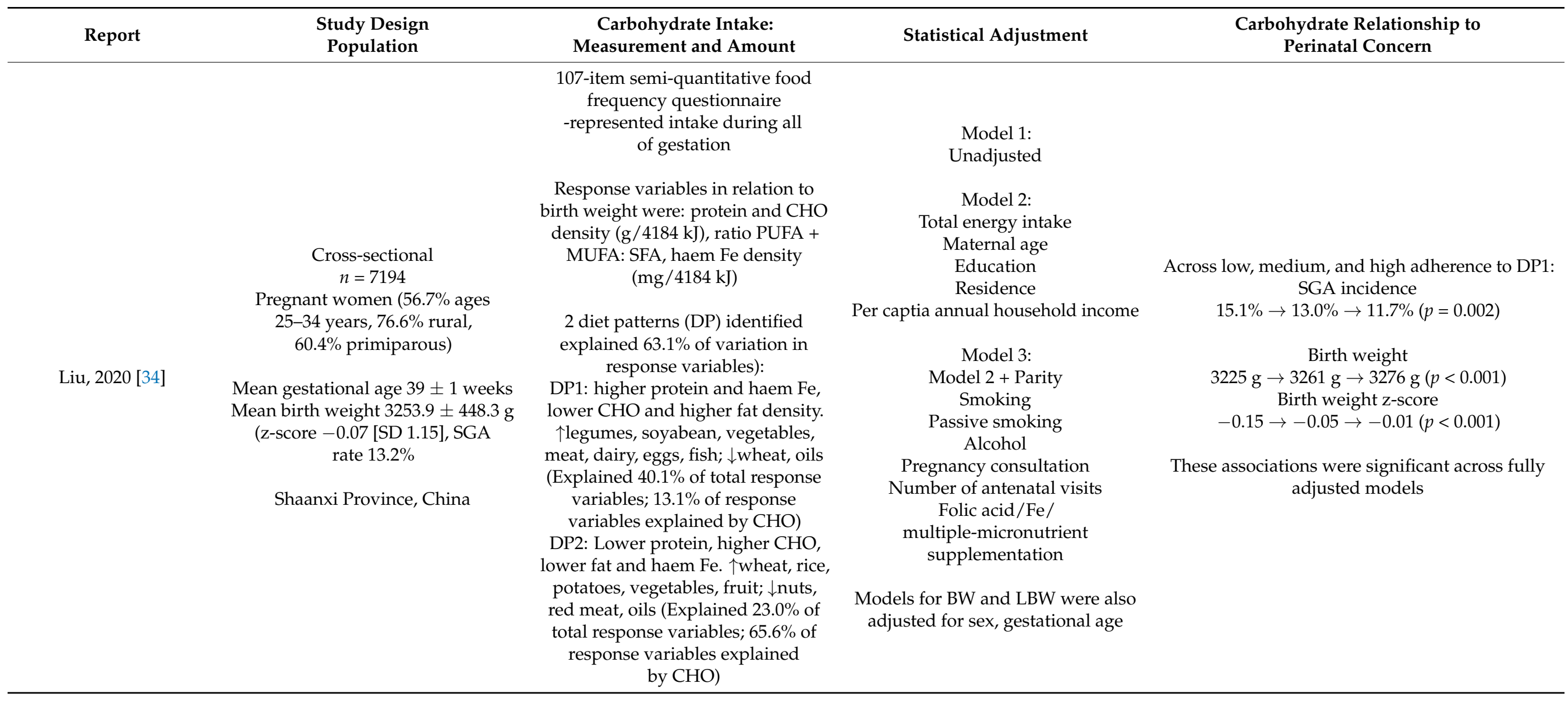


Table 1. Cont.

\begin{tabular}{|c|c|c|c|c|}
\hline Report & $\begin{array}{l}\text { Study Design } \\
\text { Population }\end{array}$ & $\begin{array}{c}\text { Carbohydrate Intake: } \\
\text { Measurement and Amount }\end{array}$ & Statistical Adjustment & $\begin{array}{c}\text { Carbohydrate Relationship to } \\
\text { Perinatal Concern }\end{array}$ \\
\hline Powell, 2020 [35] & $\begin{array}{c}\text { Observational birth cohort } \\
n=141 \text { mother/infant dyads } \\
\text { Maasai pastoralist women } \\
n=102 \text { neonates from Mwanza } \\
\text { (urban/peri-urban center) } \\
\text { Included for BW comparisons only. } \\
\text { Women were not instructed to } \\
\text { reduce EI, but intake not measured } \\
\text { All births during the dry season } \\
\text { (June-September) } \\
\text { Gestational age at delivery not } \\
\text { reported } \\
\text { Ngorongoro Conservation Area } \\
\text { (NCA), Northern Tanzania }\end{array}$ & $\begin{array}{c}\text { Food frequency questionnaire } \\
\text { developed/validated for this } \\
\text { Maasai cohort } \\
\text { Administered } 2-3 \text { d postpartum on } \\
2 \text { occasions to assess early-mid } \\
\text { (T1-2) and 3rd trimester (T3) } \\
\text { pregnancy, respectively } \\
\text { Intake T1-2 } \\
\text { Total Energy: } 1601 \pm 734.19 \mathrm{kcal} / \mathrm{d} \\
\text { CHO intake T1-2: } 276.04 \mathrm{~g} / \mathrm{d}[95 \% \\
\text { CI: } 237.72-314.37] \\
76 \% \text { of total } \\
\text { Fat intake: } 43.83 \mathrm{~g} / \mathrm{d}(37.67-49.99) \\
\text { Protein: } 45.27 \mathrm{~g} / \mathrm{d}(8.69-51.86)\end{array}$ & $\begin{array}{c}\text { Adjustments: } \\
\text { Traditional birth attendant }\end{array}$ & $\begin{array}{c}\text { Intake change from T1-2 to onset of T3: } \\
\text { Total energy: } 1601 \rightarrow 799 \pm 317.59 \mathrm{kcal} / \mathrm{d} \\
\text { CHO: } 276 \rightarrow 100.27 \mathrm{~g} / \mathrm{d}(95 \% \mathrm{CI}: 62.46-138.08) \\
\text { Fat: } 43.83 \rightarrow 23.43 \mathrm{~g} / \mathrm{d}(17.38-29.48) \\
\text { Protein: } 45.27 \rightarrow 30.17 \mathrm{~g} / \mathrm{d}(23.69-36.65) \\
\text { Reductions were: } \\
\text { Total energy: }-902.35 \pm 74.94 \mathrm{kcal} \\
\text { CHO: }-175.78 \pm 13.14 \mathrm{~g}(64 \% \text { of total) } \\
\text { Fat: }-20.397 \pm 2.32 \mathrm{~g} \\
\text { Protein: }-15.099 \pm 2.47 \mathrm{~g} \\
p<0.01 \text { for all } \\
\\
\text { Birth weight and head circumference z-scores in } \\
\text { neonates from Mwanza and NCA fell below the } \\
\text { WHO standard } \\
\text { Head circumference in neonates from NCA were } \\
\text { far lower (1.7 SD) than standard }(<50 \% \text { tile at } \\
36 \text { weeks'), more so than weight }(>50 \% \text { tile at } \\
36 \text { weeks'). } 31 \% \text { had birth weight }<2500 \mathrm{~g} \text { (vs. } \\
12 \% \text { Mwanza), } 40 \% \text { were microcephalic (vs. } \\
n=2 \text { Mwanza). }\end{array}$ \\
\hline
\end{tabular}


Table 1. Cont.

\begin{tabular}{|c|c|c|c|c|}
\hline Report & $\begin{array}{l}\text { Study Design } \\
\text { Population }\end{array}$ & $\begin{array}{c}\text { Carbohydrate Intake: } \\
\text { Measurement and Amount }\end{array}$ & Statistical Adjustment & $\begin{array}{c}\text { Carbohydrate Relationship to } \\
\text { Perinatal Concern }\end{array}$ \\
\hline $\begin{array}{l}\text { Morisaki, } 2018 \text { [36] } \\
\text { (Same cohort as } \\
\text { Eshak) [30] }\end{array}$ & $\begin{array}{c}\text { Observational birth cohort } \\
n=91,637 \\
\text { Healthy pregnant women }(40.3 \% \\
\text { primiparous, } 31 \pm 5 \text { years }) \\
73.6 \% \text { had BMI } 18.5 \text { to }<25 \mathrm{~kg} / \mathrm{m}^{2} \\
\text { Gestational age at delivery: } \\
>28 \text { weeks and } \leq 42 \text { weeks } \\
\text { Mean birth weight } 3028 \pm 406 \mathrm{~g} \\
(6.9 \% \text { SGA) } \\
\text { Japan (15 regions represented) }\end{array}$ & $\begin{array}{c}\text { Food frequency questionnaire } \\
\text { Early pregnancy (FFQ1) to represent } \\
\text { previous year } \\
\text { Mid-pregnancy to represent intake } \\
\text { during pregnancy } \\
\text { Intake at FFQ1 } \\
\text { Total energy: } 7475.1 \pm 2575.7 \mathrm{~kJ} / \mathrm{d} \\
\text { CHO: } 243.4 \pm 80.2 \mathrm{~g} / \mathrm{d}(55.3 \%) \\
\text { Fat: } 59.9 \pm 28.4 \mathrm{~g} / \mathrm{d}(29.5 \%) \\
\text { Protein: } 61.2 \pm 25.6 \mathrm{~g} / \mathrm{d}(13.5 \%) \\
\\
\text { Intake at FFQ2 } \\
\text { Total energy: } 7184 \pm 2506 \mathrm{~kJ} / \mathrm{d} \\
\text { CHO: } 233.7 \pm 77 \mathrm{~g} / \mathrm{d}(55.3 \%) \\
\text { Fat: } 58.2 \pm 27.9 \mathrm{~g} / \mathrm{d}(29.8 \%) \\
\text { Protein: } 58.9 \pm 25.1 \mathrm{~g} / \mathrm{d}(13.6 \%)\end{array}$ & $\begin{array}{c}\text { For models where } \mathrm{CHO} \text { or fat were } \\
\text { used to predict fetal growth, } \\
\text { adjusted for: total energy intake } \\
\text { Protein intake } \\
\text { CHO or fat intake (appropriate } \\
\text { to model) } \\
\text { Confounders: } \\
\text { Maternal age } \\
\text { Parity } \\
\text { Education } \\
\text { Income } \\
\text { Pre-pregnancy BMI } \\
\text { Height } \\
\text { Smoking status } \\
\text { Infant sex } \\
\text { Adjustments for: recruitment site } \\
\text { Total energy intake } \\
\text { Gestational weight gain } \\
\text { Age }\end{array}$ & $\begin{array}{c}\text { FFQ1 and FFQ2 related to birth weight: } \\
\text { Birth weight was highest with } 12 \% \text { protein even } \\
\text { when isoenergetic replacement with CHO or fat } \\
\text { was modeled. Lower birth weight with } \\
\text { protein }>14 \% \\
\text { U-shaped association between protein density } \\
\text { and SGA risk. Lowest SGA risk with protein at } \\
12 \% \text { even when isoenergetic replacement with } \\
\text { CHO or fat was modeled. Higher SGA risk if } \\
\text { protein }>15 \% \\
\text { Controlled for protein, energy intake and } \\
\text { maternal characteristics: } \\
\text { Fat (FFQ1) } \\
\text { Fat density of } 25 \% \text { associated with highest birth } \\
\text { weight. Fat density }>35 \% \text { associated with lower } \\
\text { birth weight } \\
\text { CHO (FFQ1) } \\
\text { CHO density of } 59 \%(\sim 264 \mathrm{~g} / \mathrm{d}) \text { had highest birth } \\
\text { weight. CHO density }<47 \%(\sim 210 \mathrm{~g} / \mathrm{d}) \text { had lower } \\
\text { birth weight. }\end{array}$ \\
\hline
\end{tabular}


Table 1. Cont.

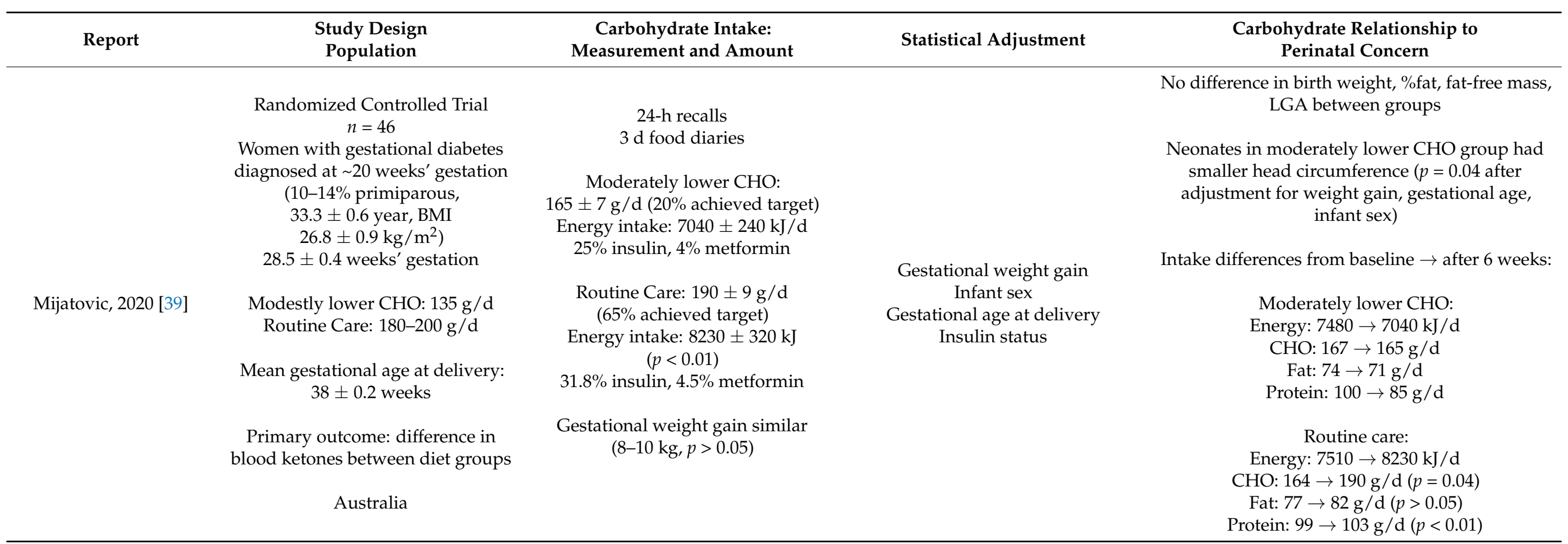


Table 1. Cont.

\begin{tabular}{|c|c|c|c|c|}
\hline Report & $\begin{array}{l}\text { Study Design } \\
\text { Population }\end{array}$ & $\begin{array}{l}\text { Carbohydrate Intake: } \\
\text { Measurement and Amount }\end{array}$ & Statistical Adjustment & $\begin{array}{c}\text { Carbohydrate Relationship to } \\
\text { Perinatal Concern }\end{array}$ \\
\hline \multirow{3}{*}{ Harreiter, 2019 [40] } & $\begin{array}{c}\text { Randomized controlled trial } \\
\text { Secondary analysis } \\
n=436\end{array}$ & $\begin{array}{l}\text { 12-item questionnaire, frequencies } \\
\text { (days/wk) } \\
\text {-Only portions recorded }\end{array}$ & Baseline level of outcome variable & \\
\hline & $\begin{array}{c}\text { Women with obesity } \\
<20 \text { weeks' gestation }(\sim 35 \% \\
\text { primiparous, } \sim 32 \pm 5 \text { years, } \\
\left.\text { Pre-pregnancy BMI } \sim 34 \pm 4 \mathrm{~kg} / \mathrm{m}^{2}\right) \\
\text { Mean gestational age at delivery } \\
\sim 39 \pm 2 \text { weeks } \\
\text { Mean birth weight } \sim 3500 \mathrm{~g}\end{array}$ & $\begin{array}{c}\text { 24-28 weeks (HE-No HE, adjusted } \\
\text { mean difference }(95 \% \mathrm{CI})) \\
\text { Portion size: }-2.8(-5.4,-0.1) * \\
\text { CHO: }-2.0(-6.4,2.3) \\
\text { Fat: }-1.3(-2.3,-0.2) \\
\text { Protein: } 1.1(-0.2,2.4)\end{array}$ & $\begin{array}{c}\text { Or } \\
\text { Baseline level of outcome variable }+ \\
\text { age }+ \text { BMI at assessment date }+ \\
\text { gestational age }+ \text { HOMA-IR }+ \\
\text { self-reported physical activity }+ \\
\text { self-reported food intake + smoking }\end{array}$ & $\begin{array}{l}\text { No differences in birth weight, LGA or SGA } \\
\text { No difference in physical activity } \\
\text { Weight gain (HE vs. No HE) } \\
\text { 24-28 weeks' gestation: } 3.3 \pm 2.7 \text { vs. } 4.3 \pm 2.8 \mathrm{~kg} \\
(p<0.001)\end{array}$ \\
\hline & $\begin{array}{c}\text { Healthy eating: } n=221 \\
\text { No healthy eating: } n=215 \\
\sim 20-22 \% \text { GDM rate/group }(p>0.05) \\
\text { Nine European countries }(86.7 \% \text { of } \\
\text { European descent) }\end{array}$ & $\begin{array}{c}\text { 35-37 weeks } \\
\text { Portion size: }-3.8(-6.8,-0.9) * \\
\text { CHO: }-6.2(-11.6,-0.9) * \\
\text { Fat: }-1.5(-2.8,-0.3) * \\
\text { Protein: } 0.3(-1.2,1.7) \\
* p<0.05 \\
* *<<0.01\end{array}$ & $\begin{array}{l}\text { Gestational weight gain analyses } \\
\text { adjusted for baseline BMI } \\
\text { Dietary, physical activity analyses } \\
\text { adjusted for baseline level }\end{array}$ & $\begin{array}{c}35-37 \text { weeks' gestation: } 7.0 \pm 4.4 \text { vs. } 8.5 \pm 4.7 \mathrm{~kg} \\
(p<0.01)\end{array}$ \\
\hline
\end{tabular}

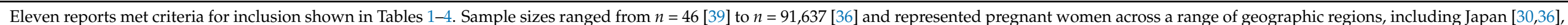

China [34], South Africa [31], India [32], Malawi [33], Tanzania [35], Australia [39], Norway [37], the Netherlands [38], and across Europe [40]. 
Recently published data reveal other patterns of carbohydrate intake related to fetal growth. A cohort study in China $(n=7194)$ [34] showed a diet pattern lowest in carbohydrate (\%EI not reported), with higher protein and fat, explained $40 \%$ of the variance in BW after adjustment for total EI and multiple confounders. BW was higher and SGA risk was lowest in those most adherent to the LC diet pattern (Table 1). Conversely, in India ( $n=1837$ ) [32], women who consumed $>70 \%$ of energy from carbohydrate had the highest SGA rate (29\%; male infants) after controlling for EI. In Malawi, carbohydrate intake $>72 \%$ was negatively associated with HC (adjusted for total EI) (Figure 1) [33]. Importantly, in the cohorts from India and Malawi, higher carbohydrate intake was accompanied by modestly lower protein intake $(11.5 \%$ and $\sim 10 \%$, respectively) [32,33]. This could be a potential factor in the SGA observations [44], although statistically protein intake was not associated with fetal growth. Collectively, the data suggest that a LC diet pattern may support appropriate fetal growth, but further studies are needed to separate the effects of LC from low EI. Moreover, carbohydrate intake as high as $70 \%$ can be a surrogate for poor diet quality, rich in foods with high glycemic index carbohydrates and added sugars [47], and the effect of diet quality remains unclear. Nonetheless, if low EI and LC intake are interlinked (as weight loss studies suggest) [41,48], caution is needed in pregnancy.

\subsection{Question 2: In Pregnant Women Who Consume a LC Diet, Is There Greater Fetal Exposure to} Maternal Ketones?

Background. Maternal ketones often increase in normal pregnancy, leading to high fetal ketone levels via passive diffusion across the placenta [49]. Maternal ketogenesis is most evident in later pregnancy due to increased lipolysis and fetal energy demand [19]. Carbohydrate restriction may also promote maternal ketonemia by increasing the ratio of glucagon to insulin, promoting oxidation of FFA to betahydroxybutyrate and other ketones. A safety concern for a LC diet in pregnancy is the potential risk of higher fetal exposure to maternal ketones. Early epidemiological studies evaluated the impact of fetal exposure to maternal ketones secondary to energy restriction, with no consistent association between ketonemia or ketonuria and poor fetal outcomes [50]. However, a seminal prospective US cohort study $(n=223$, pre-existing diabetes, GDM or normal glucose tolerance) demonstrated an inverse correlation between higher maternal third trimester betahydroxybutyrate and FFA and lower offspring intellectual development scores at 2-5 years [17]. Measures of glycemic control did not correlate with cognitive scores, but total carbohydrate and EI, and maternal body mass index $\left(\mathrm{BMI} \mathrm{kg} / \mathrm{m}^{2}\right)$ were not reported. Energy restriction (1200 $\mathrm{kcal} / \mathrm{d}$ [50\% carbohydrate, 30\% fat, 20\% protein]) in 12 women with GDM and obesity over the course of 1-week increased betahydroxybutyrate and ketonuria (vs. $2400 \mathrm{kcal} / \mathrm{d}$ diet) [20]. Ketonuria was also observed in some women consuming the control diet. In a separate study, reducing energy intake by $50 \%$ caused a 2.7 -fold increase in betahydroxybutyrate and 2-fold increase in ketonuria $(1200 \mathrm{kcal} / \mathrm{d})$ with no detectable changes with 33\% energy restriction (1600-1800 kcal/d) [21]; however, fetal outcomes were not reported. Despite the limitations of the historical data, fetal exposure to maternal ketones remains a safety concern in pregnancy.

Review. In a secondary analysis of a multi-site HE RCT [40] (Table 2), lower carbohydrate portions were associated with higher betahydroxybutyrate $(0.082 \mathrm{vs} .0 .068 \mathrm{mmol} / \mathrm{L}$; $p<0.05)$ and higher fasting glucose ( $4.7 \mathrm{vs} .4 .6 \mathrm{mmol} / \mathrm{L} ; p<0.05)$ at $24-28$ weeks' gestation (after 4-8 weeks of HE), but not at 35-37 weeks' gestation. As expected, carbohydrate intake at 24-28 weeks' gestation was negatively correlated with betahydroxybutyrate. However, an RCT of LC intake ( $165 \mathrm{~g} / \mathrm{d})$ vs. routine care $(190 \mathrm{~g} / \mathrm{d})$ (Table 2) in 46 women with GDM reported no increase in maternal betahydroxybutyrate levels over 6 weeks [39]. However, only $20 \%$ of women in the LC group achieved the prescribed $135 \mathrm{~g} / \mathrm{d}$ target despite a lower EI (7040 vs. $8230 \mathrm{~kJ})$. Thus, the effects of a LC intake $<165 \mathrm{~g} / \mathrm{d}$ in GDM on maternal ketonemia and fetal outcomes remain unclear. 
Table 2. Reports relevant to research question 2: In pregnant women who consume a LC diet, is there greater fetal exposure to maternal ketones?

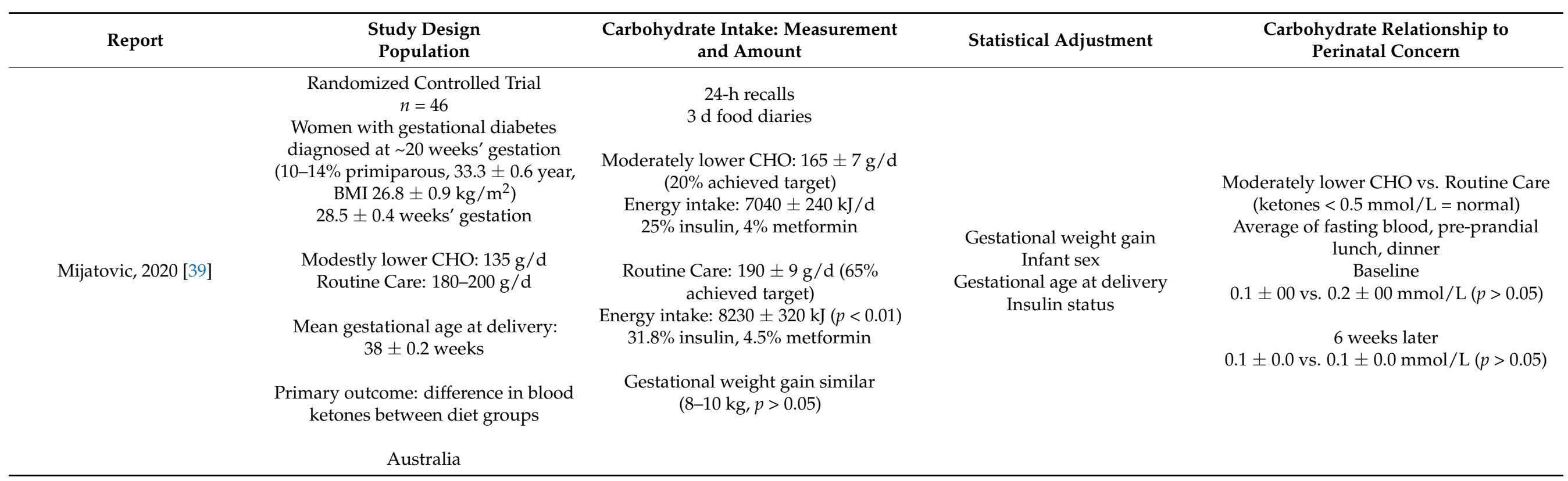


Table 2. Cont.

\begin{tabular}{|c|c|c|c|c|}
\hline Report & $\begin{array}{l}\text { Study Design } \\
\text { Population }\end{array}$ & $\begin{array}{c}\text { Carbohydrate Intake: Measurement } \\
\text { and Amount }\end{array}$ & Statistical Adjustment & $\begin{array}{c}\text { Carbohydrate Relationship to } \\
\text { Perinatal Concern }\end{array}$ \\
\hline Harreiter, 2019 [40] & $\begin{array}{c}\text { Randomized controlled trial } \\
\text { Secondary analysis } \\
n=436 \\
\text { Women with obesity } \\
<20 \text { weeks' gestation }(\sim 35 \% \\
\text { primiparous, } \sim 32 \pm 5 \text { years, } \\
\left.\text { Pre-pregnancy BMI } \sim 34 \pm 4 \mathrm{~kg} / \mathrm{m}^{2}\right) \\
\text { Mean gestational age at delivery } \\
\sim 39 \pm 2 \text { weeks } \\
\text { Mean birth weight } \sim 3500 \mathrm{~g} \\
\text { Healthy eating }(\mathrm{HE}): n=221 \\
\text { No healthy eating: } n=215 \\
\sim 20-22 \% \text { GDM rate/group }(p>0.05) \\
\text { Nine European countries }(86.7 \% \text { of } \\
\text { European descent })\end{array}$ & $\begin{array}{c}\text { 12-item questionnaire, frequencies } \\
\text { (days/wk) } \\
\text {-Only portions recorded } \\
\text { 24-28 weeks (HE-No HE, adjusted } \\
\text { mean difference }(95 \% \mathrm{CI})) \\
\text { Portion size: }-2.8(-5.4,-0.1)^{*} \\
\text { CHO: }-2.0(-6.4,2.3) \\
\text { Fat: }-1.3(-2.3,-0.2) \\
\text { Protein: } 1.1(-0.2,2.4) \\
\\
\text { 35-37 weeks' gestation } \\
\text { Portion size: }-3.8(-6.8,-0.9) * * \\
\text { CHO: }-6.2(-11.6,-0.9) * \\
\text { Fat: }-1.5(-2.8,-0.3) * \\
\text { Protein: } 0.3(-1.2,1.7) \\
* p<0.05 \\
* *<<0.01\end{array}$ & $\begin{array}{l}\text { Baseline level of outcome variable } \\
\text { Or } \\
\text { Baseline level of outcome variable } \\
+ \text { age + BMI at assessment date + } \\
\text { gestational age + HOMA-IR + } \\
\text { self-reported physical activity + } \\
\text { self-reported food intake + } \\
\text { smoking } \\
\text { Gestational weight gain analyses } \\
\text { adjusted for baseline BMI } \\
\text { Dietary, physical activity analyses } \\
\text { adjusted for baseline level }\end{array}$ & $\begin{array}{c}\text { HE vs. No HE } \\
24-28 \text { weeks' gestation } \\
\text { Fasting blood beta-hydroxybutyrate: } \\
0.082 \pm 0.065 \text { vs. } 0.068 \pm 0.067(p<0.05) \\
35-37 \text { weeks' gestation } \\
\text { Fasting blood beta-hydroxybutyrate: } \\
0.107 \pm 0.071 \text { vs. } 0.101 \pm 0.092\end{array}$ \\
\hline
\end{tabular}


3.3. Question 3: Do Pregnant Women Who Consume a LC Diet Have an Increased Risk for Micronutrient Deficiency?

Background. Recent cohort studies suggest maternal micronutrient intakes are commonly below recommended guidelines [37-39]. Maternal micronutrient deficiency may contribute to adverse fetal development and chronic disease via direct effects on hormonal adaptation and epigenetic gene regulation [51]. The potential for a LC diet to magnify micronutrient deficiency is a commonly cited concern. In the pre-conception period, a restricted carbohydrate intake (defined as $\leq 5$ th percentile among a control population, $\sim 95 \mathrm{~g} / \mathrm{d}$ ) has been associated with neural tube defects [10], potentially independent of folic acid deficiency [11].

Review. An RCT conducted in women with GDM showed compared to a routine care diet containing $190 \mathrm{~g} / \mathrm{d}$ of carbohydrate, a LC diet (165 g/d) achieved lower iron and iodine intakes from food (iron: $8.7 \pm 0.4$ vs. $10.6 \pm 0.4 \mathrm{mg} / \mathrm{d}, p<0.01$; iodine: $147 \pm 11 \mathrm{vs}$. $196 \pm 14 \mu \mathrm{g} / \mathrm{d}, p<0.01$ ) (Table 3, Figure 1) [39]. Both iron and iodine decreased from pre- to post-study intervention in the LC group, suggesting a LC intake may promote an increased risk of micronutrient deficiency, particularly without supplement use. In a large Danish study [37], 44\% of pregnant women reported carbohydrate intake below the recommended intake (RI) of 45-60\% EI. Inadequate micronutrient intakes of folate (54\% of women), iron $(50 \%)$, calcium $(36 \%)$, vitamin D $(29 \%)$, iodine $(24 \%)$ and selenium (41\%) were also reported. However, whether the women with carbohydrate intake below the RI had higher incidence of micronutrient deficiencies was unknown. A separate study in Dutch women $(n=105)$ consuming moderate amounts of carbohydrate (range 43.2-49.7\%) [38], showed that iron intake was inversely associated with glucose status. Folate, vitamins B6 and D intakes significantly changed through pregnancy, but were explained by supplement use rather than carbohydrate intake. In Japan $(n=78,793)$, median carbohydrate intake was 55\% of EI, with most women consuming less than the Japanese recommendation (57.5\% EI) [30]. Micronutrient intakes below recommendations were also reported (Table 3), suggesting micronutrient deficiencies may exist even with higher carbohydrate intake. Ponderal index was reduced across increasing quartiles of most micronutrients. Taken together, studies suggest micronutrient deficiency is common in pregnant women, and may be independent of dietary carbohydrate intake. 
Table 3. Reports relevant to research question 3: Do pregnant women who consume a LC diet have an increased risk for micronutrient deficiency?

\begin{tabular}{|c|c|c|c|c|}
\hline Report & $\begin{array}{l}\text { Study Design } \\
\text { Population }\end{array}$ & $\begin{array}{c}\text { Carbohydrate Intake: Measurement } \\
\text { and Amount }\end{array}$ & Statistical Adjustment & $\begin{array}{l}\text { Carbohydrate Relationship to } \\
\text { Perinatal Concern }\end{array}$ \\
\hline Eshak, 2020 [30] & $\begin{array}{l}\text { Observational birth cohort } \\
\quad n=78,793 \\
\text { Healthy pregnant women }(39.6 \% \\
\text { primiparous, } 31 \pm 5 \text { years }) \\
78 \% \text { had BMI } 18.5 \text { to }<25 \mathrm{~kg} / \mathrm{m}^{2} \\
\text { Mean gestational age at delivery } \\
38.9 \pm 1.5 \text { weeks } \\
\text {-Mean birth weight reported in } \\
\text { right column } \\
\text { Japan (15 regions represented) }\end{array}$ & $\begin{array}{c}\text { Food frequency questionnaire Trimester } 2 \\
\text { Median (IQR) CHO intake } 223.8 \\
(182.6-272.4) \mathrm{g} / \mathrm{d} \\
55.3 \% \text { of total energy intake } \\
\\
\text { 61.9\% of women consumed } \\
\text { <recommended amount of CHO/d }(57.5 \%) \\
\text { Quartiles of CHO } \% \text { Intake } \\
\text { Q1: } 45.1 \%(1075 \mathrm{kcal} / \mathrm{d}) \\
\text { Q2: } 52.9 \%(1466 \mathrm{kcal} / \mathrm{d}) \\
\text { Q3: } 57.7 \%(1800 \mathrm{kcal} / \mathrm{d}) \\
\text { Q4: } 64.9 \%(2650 \mathrm{kcal} / \mathrm{d}) \\
\\
\\
\text { Proportion of women consumed }< \\
\text { recommended amount of micronutrients } \\
\text { Vitamin A } 63 \% \\
\text { Vitamin K } 48 \% \\
\text { Vitamin E } 61 \% \\
\text { Vitamin D } 87 \% \\
\text { Vitamin C } 67 \%, \\
\text { Vitamin B6 } 73 \% \\
\text { Folate } 88 \% \\
\text { Vitamin B12 } 26 \%\end{array}$ & $\begin{array}{c}\text { Geometric means of nutrients adjusted for: } \\
\text { Maternal age } \\
\text { Height } \\
\text { Education } \\
\text { Household income } \\
\text { Pre-pregnancy BMI } \\
\text { Net weight change in pregnancy } \\
\text { Smoking } \\
\text { Alcohol } \\
\text { Thyroid disease } \\
\text { Use of folate supplement } \\
\text { Offspring sex, gestational age at delivery }\end{array}$ & $\begin{array}{c}\text { Increasing quartiles of micronutrients: } \\
\text { Vitamin C and folate intake associated with } \\
\text { birthweight; Vitamins C, D, K, B6, B12 and folate } \\
\text { associated with birth length } \\
\text { Vitamins A, E and D associated with head } \\
\text { circumference; Vitamins A, C and D associated } \\
\text { with chest circumference } \\
\text { Vitamin K inversely associated with the ponderal } \\
\text { index in the offspring }\end{array}$ \\
\hline
\end{tabular}


Table 3. Cont

\begin{tabular}{|c|c|c|c|c|}
\hline Report & $\begin{array}{l}\text { Study Design } \\
\text { Population }\end{array}$ & $\begin{array}{c}\text { Carbohydrate Intake: Measurement } \\
\text { and Amount }\end{array}$ & Statistical Adjustment & $\begin{array}{c}\text { Carbohydrate Relationship to } \\
\text { Perinatal Concern }\end{array}$ \\
\hline Saunders, 2019 [37] & $\begin{array}{c}\text { Observational birth cohort } \\
n=1674 \\
\text { Healthy pregnant women } \\
(62.9 \% \text { primiparous, } 32.5 \pm 4.1 \text { years }) \\
\text { BMI } 24.6 \pm 3.5 \mathrm{~kg} / \mathrm{m}^{2} \\
\text { Recruited between } 16-22 \text { weeks' gestation } \\
\text { Norway }\end{array}$ & $\begin{array}{c}\text { Food frequency questionnaire } \\
\text { First half of pregnancy } \\
\text { Total energy: } 10,082(4139) \mathrm{kJ} \\
\text { CHO: } 45.7(42.3-49.2) \% \\
\text { Fat: } 34.5(31.2-37.8) \% \\
\text { Protein: } 16.5(15.1-18.1) \% \\
\\
\text { Below and above Recommended Intake } \\
\text { Range for macronutrients: } \\
\text { CHO: Below } 43.9 \% \text {, above } 0.5 \% \\
\text { Fat: Below } 2.9 \% \text {, above } 14.0 \% \\
\text { Protein: Below } 0.2 \% \text {, above } 6.9 \% \\
\text { Micronutrients: } \\
\text { Vit A: Below } 9.6 \% \text {, above } 90.4 \% \\
\text { Vit C: Below } 4.4 \% \text {, above } 95.6 \% \\
\text { Vit D: Below } 28.7 \% \text {, above } 71.3 \% \\
\text { Vit B12: Below } 0.3 \% \text {, above } 99.7 \% \\
\text { Iodine: Below } 24.4 \% \text {, above } 75.6 \% \\
\text { Folate: Below } 54.4 \text {, above } 45.6 \% \\
\text { Zinc: Below } 10.2, \text { above } 89.8 \% \\
\text { Ca: Below } 36.2 \% \text {, above } 63.8 \% \\
\text { Selenium: Below } 41.3 \% \text {, above } 58.7 \% \\
\text { Iron: Below } 41.3 \% \text {, above } 58.7 \% \\
\text { Median (IQRR) } \\
\text { Based on Nordic Nutrition } \\
\text { Recommendations, 2012 [52] }\end{array}$ & Educational level (post-hoc analysis) & $\begin{array}{l}\text { No association between educational levels and } \\
\text { micronutrient intake }\end{array}$ \\
\hline
\end{tabular}


Table 3. Cont

\begin{tabular}{|c|c|c|c|c|}
\hline Report & $\begin{array}{l}\text { Study Design } \\
\text { Population }\end{array}$ & $\begin{array}{l}\text { Carbohydrate Intake: Measurement } \\
\text { and Amount }\end{array}$ & Statistical Adjustment & $\begin{array}{c}\text { Carbohydrate Relationship to } \\
\text { Perinatal Concern }\end{array}$ \\
\hline Looman, 2019 [38] & $\begin{array}{c}\text { Observational Birth Cohort } \\
n=105 \\
\text { Preconception to }<24 \text { weeks' gestation } \\
\text { (32yo, } 93 \% \text { multiparous, median BMI } \\
\text { preconception } 24.4 \mathrm{~kg} / \mathrm{m}^{2} \\
\text { The Netherlands }\end{array}$ & $\begin{array}{c}\text { FFQ } \\
75 \mathrm{~g} 2-\mathrm{h} \text { OGTT } \\
\text { At pre-conception, } 12 \text { and } 24 \text { weeks' } \\
\text { gestation } \\
\text { Energy intake increased during pregnancy } \\
\text { from } 8583(6713 ; 9462) \mathrm{kJ} \text { at preconception } \\
\text { to } 9189(7432 ; 10,541) \mathrm{kJ} \text { at } 24 \text { weeks' } \\
\text { gestation } \\
\text { Median CHO } 46.5 \%(43.2 ; 49.7) \\
\text { Preconception } 45.4 \%(42.3 ; 48.6) \\
\text { TM1 46.5\% }(45.2 ; 50.3) \\
\text { TM2 48.1\% }(44.8 ; 50.3) \\
\end{array}$ & $\begin{array}{c}\text { Covariates: } \\
\text { Age } \\
\text { Education } \\
\text { Ethnicity } \\
\text { Parity } \\
\text { Smoking } \\
\text { Nausea in pregnancy } \\
\text { Vomiting in pregnancy } \\
\text { Season of blood collection } \\
\text { Physical Activity } \\
\text { Energy intake } \\
\text { Alcohol } \\
\text { Time between measurements } \\
\text { Hx of GDM } \\
\text { BMI } \\
\text { Adjusted for supplement intake }\end{array}$ & $\begin{array}{l}\text { Iron intake inversely associated with fasting } \\
\text { glucose and HbA1c } \\
\text { Folate, vitamin B6 and vitamin D levels } \\
\text { significantly changed through pregnancy, } \\
\text { accounted for by intake of supplements }\end{array}$ \\
\hline Mijatovic, 2020 [39] & $\begin{array}{c}\text { Randomized Controlled Trial } \\
n=46 \\
\text { Women with gestational diabetes } \\
\text { diagnosed at } \sim 20 \text { weeks' gestation }(10-14 \% \\
\text { primiparous, } 33.3 \pm 0.6 \text { years, BMI } \\
\left.26.8 \pm 0.9 \mathrm{~kg} / \mathrm{m}^{2}\right) \\
28.5 \pm 0.4 \text { weeks' gestation } \\
\text { Modestly lower CHO: } 135 \mathrm{~g} / \mathrm{d} \\
\text { Routine Care: } 180-200 \mathrm{~g} / \mathrm{d} \\
\text { Mean gestational age at delivery: } \\
38 \pm 0.2 \text { weeks' } \\
\text { Primary outcome: difference in blood } \\
\text { ketones between diet groups } \\
\text { Australia }\end{array}$ & $\begin{array}{c}\text { 24-h recalls } \\
\text { 3d food diaries } \\
\\
\text { Moderately lower CHO: } 165 \pm 7 \mathrm{~g} / \mathrm{d} \\
\text { (20\% achieved target) } \\
\text { Energy intake: } 7040 \pm 240 \mathrm{~kJ} / \mathrm{d} \\
25 \% \text { insulin, } 4 \% \text { metformin } \\
\\
\text { Routine Care: } 190 \pm 9 \mathrm{~g} / \mathrm{d} \\
(65 \% \text { achieved target }) \\
\text { Energy intake: } 8230 \pm 320 \mathrm{~kJ}(p<0.01) \\
31.8 \% \text { insulin, } 4.5 \% \text { metformin } \\
\text { Gestational weight gain similar }(8-10 \mathrm{~kg}, \\
p>0.05)\end{array}$ & $\begin{array}{l}\text { Gestational weight gain } \\
\text { Infant sex } \\
\text { Gestational age at delivery } \\
\text { Insulin status }\end{array}$ & Moderately lower $\mathrm{CHO}$ : lower $\mathrm{Fe}$, iodine \\
\hline
\end{tabular}


3.4. Question 4: Do Pregnant Women Who Consume a LC Diet Have Higher TG or FFA, Increasing Fetal Exposure to Lipids?

Background. While glucose is a dominant substrate for fetal growth, mounting evidence suggests a positive association between maternal lipids (TG, FFA) and fetal overgrowth [53]. Indeed, some reports demonstrate a more robust association between maternal TG and fetal growth than maternal glucose [54]. At the Fifth International Workshop on GDM in 2005, nutrition therapy recommendations focused only on carbohydrate restriction were revoked [25]. However, the role of maternal lipids in fetal growth remains unclear [55] despite the association of maternal FFA and fetal overgrowth [24,53]. In addition to a reduction in carbohydrate content, low carbohydrate diets also typically increase and have relatively higher proportions of daily caloric intake from fats (55-65\%) and/or protein (25-30\%) [56]. Specific to pregnancy, there is concern that higher dietary fat intake could increase maternal TG and FFA, potentially increasing fetal exposure to maternal lipids secondary to heightened maternal insulin resistance, leading to overgrowth [57]. Fetal exposure to increased maternal lipids, particularly through high fat diets, has been linked with developmental programming of offspring obesity and fatty liver in animal models and in human cohorts [44,57-61].

Review. In a secondary analysis of a multi-site RCT (Table 4) [40], women in the lower carbohydrate portion group (vs. higher carbohydrate) had higher fasting FFA and glucose after 4-8 weeks of intervention. At 35-37 weeks' gestation, only FFA remained higher. At 24-28 weeks' gestation, weak negative associations between carbohydrate intake and fasting FFA $(\mathrm{r}=-0.12, p<0.03)$ and fasting glucose $(\mathrm{r}=-0.11, p<0.03)$ were reported. There were no differences in cord blood C-peptide or fetal growth, and maternal lipids were not correlated with fetal growth. Future investigations are needed to establish the effects of higher fat intake on maternal TG/FFA concentrations and fetal growth. 
Table 4. Reports relevant to research question 4: Do pregnant women who consume a LC diet have higher TG or FFA, increasing fetal exposure to lipids?

\begin{tabular}{|c|c|c|c|c|}
\hline Report & $\begin{array}{l}\text { Study Design } \\
\text { Population }\end{array}$ & $\begin{array}{l}\text { Carbohydrate Intake: } \\
\text { Measurement and Amount }\end{array}$ & Statistical Adjustment & $\begin{array}{c}\text { Carbohydrate Relationship to Perinatal } \\
\text { Concern }\end{array}$ \\
\hline \multirow{3}{*}{ Harreiter, 2019 [40] } & $\begin{array}{c}\text { Randomized controlled trial } \\
\text { Secondary analysis } \\
n=436\end{array}$ & $\begin{array}{c}\text { 12-item questionnaire, frequencies } \\
\text { (days/wk) } \\
\text {-Only portions recorded }\end{array}$ & Baseline level of outcome variable & \\
\hline & $\begin{array}{c}\text { Women with obesity } \\
<20 \text { weeks' gestation }(\sim 35 \% \\
\text { primiparous, } \sim 32 \pm 5 \text { years, } \\
\left.\text { pre-pregnancy BMI } \sim 34 \pm 4 \mathrm{~kg} / \mathrm{m}^{2}\right) \\
\text { Mean gestational age at delivery } \\
\sim 39 \pm 2 \text { weeks } \\
\text { Mean birth weight } \sim 3500 \mathrm{~g}\end{array}$ & $\begin{array}{l}\text { 24-28 weeks' gestation }(\mathrm{HE}-\mathrm{No} \\
\text { HE, adjusted mean difference } \\
(95 \% \mathrm{CI})) \\
\text { Portion size: }-2.8(-5.4,-0.1) * \\
\text { CHO: }-2.0(-6.4,2.3) \\
\text { Fat: }-1.3(-2.3,-0.2) \\
\text { Protein: } 1.1(-0.2,2.4)\end{array}$ & $\begin{array}{c}\text { Or } \\
\text { Baseline level of outcome variable }+ \\
\text { age + BMI at assessment date }+ \\
\text { gestational age + HOMA-IR }+ \\
\text { self-reported physical activity }+ \\
\text { self-reported food intake + smoking }\end{array}$ & $\begin{array}{c}\text { HE vs. No HE } \\
24-28 \text { weeks' gestation } \\
\text { TG: } 1.88 \pm 0.63 \text { vs. } 1.85 \pm 0.68 \mathrm{mmol} / \mathrm{L} \\
\text { FFA: } 0.60 \pm 0.19 \text { vs. } 0.55 \pm 0.17 \mathrm{mmol} / \mathrm{L} \\
(p<0.01) \\
\text { Fasting glucose: } 4.8 \pm 0.4 \text { vs. } 4.6 \pm 0.4 \mathrm{mmol} / \mathrm{L} \\
(p<0.05)\end{array}$ \\
\hline & $\begin{array}{c}\text { Healthy eating: } n=221 \\
\text { No healthy eating: } n=215 \\
\sim 20-22 \% \text { GDM rate/group }(p>0.05) \\
\text { Nine European countries }(86.7 \% \text { of } \\
\text { European descent) }\end{array}$ & $\begin{array}{c}\text { 35-37 weeks' gestation } \\
\text { Portion size: }-3.8(-6.8,-0.9) * * \\
\text { CHO: }-6.2(-11.6,-0.9)^{*} \\
\text { Fat: }-1.5(-2.8,-0.3)^{*} \\
\text { Protein: } 0.3(-1.2,1.7) \\
{ }^{*} p<0.05 \\
* * p<0.01\end{array}$ & $\begin{array}{l}\text { Gestational weight gain analyses } \\
\text { adjusted for baseline BMI } \\
\text { Dietary, physical activity analyses } \\
\text { adjusted for baseline level }\end{array}$ & $\begin{array}{c}\text { 35-37 weeks' gestation } \\
\text { TG } 2.42 \pm 0.8 \text { vs. } 2.27 \pm 0.8 \mathrm{mmol} / \mathrm{L} \\
\text { FFA: } 0.64 \pm 0.23 \text { vs. } 0.59 \pm 0.21(p<0.05) \\
\text { Fasting glucose: } 4.6 \pm 0.5 \text { vs. } 4.5 \pm 0.4 \mathrm{mmol} / \mathrm{L}\end{array}$ \\
\hline
\end{tabular}




\section{Conclusions}

Guided by four questions based on safety considerations related to LC diets during pregnancy, we set out to systematically evaluate recently published evidence to identify an acceptable LC intake threshold in pregnancy (Figure 1), if apparent. Available evidence suggests an optimal carbohydrate range between $47-70 \%$ EI supports normal fetal growth. Importantly, both the lower carbohydrate threshold of $47 \%$ EI, supported by data from a study of nearly 100,000 women [36], and the upper carbohydrate threshold of $70 \%$ EI [32,33], were independent of total EI. The lower threshold is higher than the conventionally recognized LC threshold of $33-40 \%$ EI in pregnancy [4]. Across studies, fetal growth tracked consistently with carbohydrate intake such that BWs are lower and incidences of SGA are higher with lower carbohydrate intake. While several studies did not control for total EI $[30,31,35]$, the same response was observed in the large cohort study from Japan which controlled for EI [36]. The effects of a carbohydrate intake below the IOM recommendation of $175 \mathrm{~g} / \mathrm{d}$ remain unclear, particularly intakes below the threshold at which women may experience ketonemia $(<50 \mathrm{~g} / \mathrm{d}$ outside of pregnancy) $[39,62,63]$. A major confounder in studies is the concurrent reduction in total EI with LC intake, a factor that independently impairs fetal growth [43] and promotes maternal ketonemia [20]. Caution should therefore be applied because inadvertent adverse effects of LC with or without caloric restriction could be severe. For example, an in vitro study of trophoblasts cultured from first trimester chorionic villi demonstrated that ketones suppress trophoblast uptake of glucose, jeopardizing glucose transfer across the placenta [18]. Evidence evaluated here showed reductions in head circumference occurred following $~ 50 \%$ maternal energy restriction with $100 \mathrm{~g}$ carbohydrate/d [35].

The reported high prevalence of poor maternal micronutrient intake may be independent of carbohydrate intake, although LC diets $(<165 \mathrm{~g} / \mathrm{d})$ may exacerbate existing deficiencies [39]. Supplementation remains a key determinant of achieving sufficient micronutrient levels but may not always be accessible. The impact of a LC high-fat diet on maternal TG independent of the TG-raising effects of placental estrogen remains unclear [57]. Moreover, gestational weight gain is a strong independent predictor of fetal growth, requiring consideration along with carbohydrate and EI.

This systematic review and analysis of contemporary data had several strengths and limitations. Women enrolled across the 11 studies provided wide geographic representation of ethnicities in both developed and less developed countries. Further, sample sizes in the cohort studies were large and the analyses robust, with adequate control for confounding variables in most cases. Unfortunately, maternal glucose and ketone concentrations were not reported in relation to carbohydrate intake in the large cohort studies, and indicators of diet quality were lacking. It was also not possible to consider the effect of maternal obesity; in fact, many of the women were of normal weight across studies (Tables 1-4). Although several studies included women who consumed $~ 40 \%$ of EI from carbohydrate [37-39], studies with the largest samples included women in higher carbohydrate intake ranges. No studies included women who consumed a very low carbohydrate diet (Figure 1). Although in most of the studies protein intake was not related to fetal growth, higher and lower intakes are known to be related with growth restriction [44]; future studies are required to evaluate the effects of a LC diet with higher protein intake. Optimal protein and fat intake in pregnancy were not the focus of this review per se. Explicit evaluation of the independent effects of glucose load and GI on maternal and fetal outcomes is also an important area for future investigation. Finally, few studies included women with GDM, in whom nutrition therapy is first-line treatment.

In conclusion, these data suggest that a carbohydrate intake in pregnancy between $47-70 \%$ supports normal fetal growth patterns. Due to the growing number of women with and without diabetes following a LC diet before and during pregnancy, well-controlled prospective RCTs and dose response studies examining the effects of energy-balanced dietary patterns with varying carbohydrate levels and specifically LC diets are clearly overdue. 
Author Contributions: Conceptualization, A.S., T.L.H. and J.B.-M.; Methodology, A.S., T.L.H.; Software, A.S., and T.L.H.; Validation A.S., T.L.H. and J.B.-M.; Formal Analysis, A.S., T.L.H., J.B.-M., J.M., G.D.B. and G.P.R.; Investigation, A.S., T.L.H., J.B.-M., J.M., G.D.B. and G.P.R.; Resources, A.S., J.M., T.L.H., J.B.-M., G.D.B.; Data Curation, A.S., T.L.H., J.B.-M.; Writing-Original Draft Preparation, A.S., T.L.H. and J.B.-M.; Writing-Review and Editing, A.S., T.L.H., J.B.-M., J.M., G.D.B., T.P.M. and G.P.R.; Visualization, A.S., T.L.H.; Supervision, T.L.H.; Project Administration A.S. and T.L.H.; Funding Acquisition A.S. and T.L.H. All authors have read and agreed to the published version of the manuscript.

Funding: This work was supported by the National Institutes of Health, National Institute of Diabetes and Digestive and Kidney Disease (NIH/NIDDK), grant R01DK101659 and by the National Health and Medical Research Council (NHMRC), grant GNT1148952.

Conflicts of Interest: The authors declare no conflict of interest.

\section{References}

1. Yamamoto, J.M.; Kellett, J.E.; Balsells, M.; García-Patterson, A.; Hadar, E.; Solà, I.; Gich, I.; Van Der Beek, E.M.; CastañedaGutiérrez, E.; Heinonen, S.; et al. Gestational diabetes mellitus and diet: A systematic review and meta-analysis of randomized controlled trials examining the impact of modified dietary interventions on maternal glucose control and neonatal birth weight. Diabetes Care 2018, 41, 1346-1361. [CrossRef]

2. Hernandez, T.L.; Mande, A.; Barbour, L.A. Nutrition therapy within and beyond gestational diabetes. Diabetes Res. Clin. Pract. 2018, 145, 39-50. [CrossRef] [PubMed]

3. Han, S.; Middleton, P.; Shepherd, E.; Van Ryswyk, E.; Crowther, C.A. Different types of dietary advice for women with gestational diabetes mellitus. Cochrane Database Syst. Rev. 2017, 2, CD009275. [CrossRef]

4. Jovanovic-Peterson, L.; Peterson, C.M. Dietary manipulation as a primary treatment strategy for pregnancies complicated by diabetes. J. Am. Coll. Nutr. 1990, 9, 320-325. [CrossRef] [PubMed]

5. Hernandez, T.L.; Van Pelt, R.E.; Anderson, M.A.; Daniels, L.J.; West, N.A.; Donahoo, W.; Friedman, J.E.; Barbour, L.A. A higher-complex carbohydrate diet in gestational diabetes mellitus achieves glucose targets and lowers postprandial lipids: A randomized crossover study. Diabetes Care 2014, 37, 1254-1262. [CrossRef] [PubMed]

6. Hernandez, T.L.; Van Pelt, R.E.; Anderson, M.A.; Reece, M.S.; Reynolds, R.M.; De La Houssaye, B.A.; Heerwagen, M.; Donahoo, W.; Daniels, L.J.; Chartier-Logan, C.; et al. Women with gestational diabetes mellitus randomized to a higher-complex carbohydrate/low-fat diet manifest lower adipose tissue insulin resistance, inflammation, glucose, and free fatty acids: A pilot study. Diabetes Care 2016, 39, 39-42. [CrossRef]

7. Asemi, Z.; Tabassi, Z.; Samimi, M.; Fahiminejad, T.; Esmaillzadeh, A. Favourable effects of the Dietary Approaches to Stop Hypertension diet on glucose tolerance and lipid profiles in gestational diabetes: A randomised clinical trial. Br. J. Nutr. 2013, 109, 2024-2030. [CrossRef] [PubMed]

8. Asemi, Z.; Samimi, M.; Tabassi, Z.; Esmaillzadeh, A. The effect of DASH diet on pregnancy outcomes in gestational diabetes: A randomized controlled clinical trial. Eur. J. Clin. Nutr. 2014, 68, 490-495. [CrossRef] [PubMed]

9. Institute of Medicine. Dietary Reference Intakes for Energy, Carbohydrate, Fiber, Fat, Fatty Acids, Cholesterol, Protein, and Amino Acids; The National Academies Press: Washington, DC, USA, 2005.

10. Desrosiers, T.A.; Siega-Riz, A.M.; Mosley, B.S.; Meyer, R.E. National Birth Defects Prevention Study Low carbohydrate diets may increase risk of neural tube defects. Birth Defects Res. 2018, 110, 901-909. [CrossRef]

11. Shaw, G.M.; Yang, W. Women's periconceptional lowered carbohydrate intake and NTD-affected pregnancy risk in the era of prefortification with folic acid. Birth Defects Res. 2019, 111, 248-253. [CrossRef]

12. Saeed, A.; Raana, T.; Saeed, A.M.; Humayun, A. Effect of antenatal depression on maternal dietary intake and neonatal outcome: A prospective cohort. Nutr. J. 2016, 15, 64. [CrossRef] [PubMed]

13. Ásbjörnsdóttir, B.; Ronneby, H.; Vestgaard, M.; Ringholm, L.; Nichum, V.L.; Jensen, D.M.; Raben, A.; Damm, P.; Mathiesen, E.R. Lower daily carbohydrate consumption than recommended by the Institute of Medicine is common among women with type 2 diabetes in early pregnancy in Denmark. Diabetes Res. Clin. Pract. 2019, 152, 88-95. [CrossRef]

14. Westman, E.C.; Yancy, W.S., Jr.; Humphreys, M. Dietary treatment of diabetes mellitus in the pre-insulin era (1914-1922). Perspect. Biol. Med. 2006, 49, 77-83. [CrossRef]

15. Battaglia, F.C.; Meschia, G. An Introduction to Fetal Physiology; Academic Press, Inc.: Cambridge, MA, USA, 1986.

16. Holme, A.M.; Roland, M.C.P.; Lorentzen, B.; Michelsen, T.M.; Henriksen, T. Placental glucose transfer: A human in vivo study. PLoS ONE 2015, 10, e0117084. [CrossRef]

17. Rizzo, T.; Metzger, B.E.; Burns, W.J.; Burns, K. Correlations between antepartum maternal metabolism and intelligence of offspring. N. Engl. J. Med. 1991, 325, 911-916. [CrossRef]

18. Shubert, P.J.; Gordon, M.C.; Landon, M.B.; Gabbe, S.G.; Kniss, D.A. Ketoacids attenuate glucose uptake in human trophoblasts isolated from first-trimester chorionic villi. Am. J. Obstet. Gynecol. 1996, 175, 56-62. [CrossRef]

19. Metzger, B.E.; Freinkel, N. Accelerated starvation in pregnancy: Implications for dietary treatment of obesity and gestational diabetes mellitus. Neonatology 1987, 51, 78-85. [CrossRef] [PubMed] 
20. Magee, M.S.; Knopp, R.H.; Benedetti, T.J. Metabolic effects of 1200-kcal diet in obese pregnant women with gestational diabetes. Diabetes 1990, 39, 234-240. [CrossRef] [PubMed]

21. Knopp, R.H.; Magee, M.S.; Raisys, V.; Benedetti, T. Metabolic effects of hypocaloric diets in management of gestational diabetes. Diabetes 1991, 40 (Suppl. 2), 165-171. [CrossRef] [PubMed]

22. Churuangsuk, C.; Kherouf, M.; Combet, E.; Lean, M. Low-carbohydrate diets for overweight and obesity: A systematic review of the systematic reviews. Obes. Rev. 2018, 19, 1700-1718. [CrossRef] [PubMed]

23. Li, M.; Sim, K.; Gordon, A.; Brand-Miller, J. The Effects of Nutrition and Micronutrients on Reproductive Success; Cambridge University Press: Cambridge, UK, 2018.

24. Schaefer-Graf, U.M.; Graf, K.; Kulbacka, I.; Kjos, S.L.; Dudenhausen, J.; Vetter, K.; Herrera, E. Maternal lipids as strong determinants of fetal environment and growth in pregnancies with gestational diabetes mellitus. Diabetes Care 2008, 31, 1858-1863. [CrossRef] [PubMed]

25. Metzger, B.E.; Buchanan, T.A.; Coustan, D.R.; De Leiva, A.; Dunger, P.D.; Hadden, D.R.; Hod, M.; Kitzmiller, J.L.; Kjos, S.L.; Oats, J.N.; et al. Summary and recommendations of the Fifth International Workshop-Conference on Gestational Diabetes Mellitus. Diabetes Care 2007, 30 (Suppl. 2), S251-S260. [CrossRef] [PubMed]

26. Merzouk, H.; Meghelli-Bouchenak, M.; Loukidi, B.; Prost, J.; Belleville, J. Impaired serum lipids and lipoproteins in fetal macrosomia related to maternal obesity. Biol. Neonate 2000, 77, 17-24. [CrossRef] [PubMed]

27. Misra, V.K.; Trudeau, S.; Perni, U. Maternal serum lipids during pregnancy and infant birth weight: The influence of prepregnancy BMI. Obesity 2011, 19, 1476-1481. [CrossRef]

28. Bomba-Opon, D.; Wielgos, M.; Szymanska, M.; Bablok, L. Effects of free fatty acids on the course of gestational diabetes mellitus. Neuro Endocrinol. Lett. 2006, 27, 277-280.

29. Welsh, J.; Lu, Y.; Dhruva, S.S.; Bikdeli, B.; Desai, N.R.; Benchetrit, L.; Zimmerman, C.O.; Mu, L.; Ross, J.; Krumholz, H.M. Age of data at the time of publication of contemporary clinical trials. JAMA Netw. Open 2018, 1, e181065. [CrossRef]

30. Eshak, E.S.; Okada, C.; Baba, S.; Kimura, T.; Ikehara, S.; Sato, T.; Shirai, K.; Iso, H.; For the Japan Environment and Children's Study Group. Maternal total energy, macronutrient and vitamin intakes during pregnancy associated with the offspring's birth size in the Japan Environment and Children's Study. Br. J. Nutr. 2020, 124, 558-566. [CrossRef]

31. Fahey, C.A.; Chevrier, J.; Crause, M.; Obida, M.; Bornman, R.; Eskenazi, B. Seasonality of antenatal care attendance, maternal dietary intake, and fetal growth in the VHEMBE birth cohort, South Africa. PLoS ONE 2019, 14, e0222888. [CrossRef]

32. Mukhopadhyay, A.; Thomas, T.; Bosch, R.J.; Dwarkanath, P.; Thomas, A.; Duggan, C.P.; Kurpad, A.V. Fetal sex modifies the effect of maternal macronutrient intake on the incidence of small-for-gestational-age births: A prospective observational cohort study. Am. J. Clin. Nutr. 2018, 108, 814-820. [CrossRef]

33. Hjertholm, K.G.; Iversen, P.O.; Holmboe-Ottesen, G.; Mdala, I.; Munthali, A.; Maleta, K.; Shi, Z.; Ferguson, E.; Kamudoni, P. Maternal dietary intake during pregnancy and its association to birth size in rural Malawi: A cross-sectional study. Matern. Child Nutr. 2018, 14, e12433. [CrossRef]

34. Liu, D.; Cheng, Y.; Mi, B.; Zeng, L.; Qu, P.; Li, S.; Zhang, R.; Qi, Q.; Wu, C.; Gao, X.; et al. Maternal dietary patterns during pregnancy derived by reduced-rank regression and birth weight in the Chinese population. Br. J. Nutr. 2020, 123, 1176-1186. [CrossRef]

35. Powell, C.D.; Wilson, W.M.; Olesaningo, G.; Manyama, M.; Jamniczky, H.; Spritz, R.; Cross, J.C.; Lukowiak, K.; Hallgrimsson, B.; Gonzalez, P.N. Lack of head sparing following third-trimester caloric restriction among Tanzanian Maasai. PLoS ONE 2020, 15, e0237700. [CrossRef]

36. Morisaki, N.; Nagata, C.; Yasuo, S.; Morokuma, S.; Kato, K.; Sanefuji, M.; Shibata, E.; Tsuji, M.; Senju, A.; Kawamoto, T.; et al. Optimal protein intake during pregnancy for reducing the risk of fetal growth restriction: The Japan Environment and Children's Study. Br. J. Nutr. 2018, 120, 1432-1440. [CrossRef]

37. Saunders, C.M.; Rehbinder, E.M.; Carlsen, K.C.L.; Gudbrandsgard, M.; Carlsen, K.-H.; Haugen, G.; Hedlin, G.; Jonassen, C.M.; Sjøborg, K.D.; Landrø, L.; et al. Food and nutrient intake and adherence to dietary recommendations during pregnancy: A Nordic mother-child population-based cohort. Food Nutr. Res. 2019, 63. [CrossRef]

38. Looman, M.; Geelen, A.; Samlal, R.A.K.; Heijligenberg, R.; Gunnewiek, J.M.T.K.; Balvers, M.G.J.; Wijnberger, L.D.E.; BrouwerBrolsma, E.M.; Feskens, E.J.M. Changes in micronutrient intake and status, diet quality and glucose tolerance from preconception to the second trimester of pregnancy. Nutrients 2019, 11, 460. [CrossRef] [PubMed]

39. Mijatovic, J.; Louie, J.C.Y.; Buso, M.E.C.; Atkinson, F.S.; Ross, G.P.; Markovic, T.P.; Brand-Miller, J.C. Effects of a modestly lower carbohydrate diet in gestational diabetes: A randomized controlled trial. Am. J. Clin. Nutr. 2020, 112, 284-292. [CrossRef] [PubMed]

40. Harreiter, J.; Simmons, D.; Desoye, G.; Corcoy, R.; Adelantado, J.M.; Devlieger, R.; Galjaard, S.; Damm, P.; Mathiesen, E.R.; Jensen, D.M.; et al. Nutritional lifestyle intervention in obese pregnant women, including lower carbohydrate intake, is associated with increased maternal free fatty acids, 3 - $\beta$-hydroxybutyrate, and fasting glucose concentrations: A secondary factorial analysis of the European multicenter, randomized controlled DALI lifestyle intervention trial. Diabetes Care 2019, 42, 1380-1389. [CrossRef]

41. Evert, A.B.; Dennison, M.; Gardner, C.D.; Garvey, W.T.; Lau, K.H.K.; MacLeod, J.; Mitri, J.; Pereira, R.F.; Rawlings, K.; Robinson, S.; et al. Nutrition therapy for adults with diabetes or prediabetes: A consensus report. Diabetes Care 2019, 42, 731-754. [CrossRef] 
42. Marconi, A.M.; Paolini, C.; Buscaglia, M.; Zerbe, G.; Battaglia, F.C.; Pardi, G. The impact of gestational age and fetal growth on the maternal-fetal glucose concentration difference. Obstet. Gynecol. 1996, 87, 937-942. [CrossRef]

43. Harding, J.E.; Johnston, B.M. Nutrition and fetal growth. Reprod. Fertil. Dev. 1995, 7, 539-547. [CrossRef] [PubMed]

44. Rodgers, A.; Sferruzzi-Perri, A.N. Developmental programming of offspring adipose tissue biology. Int. J. Obes. 2021, 45, 1170-1192. [CrossRef] [PubMed]

45. Hanson, M.A.; Gluckman, P.D. Early development conditioning of later health and disease: Physiology or pathophysiology? Physiol. Rev. 2014, 94, 1027-1076. [CrossRef]

46. Barker, D.J.; Thornburg, K.L. The obstetric origins of health for a lifetime. Clin. Obstet. Gynecol. 2013, 56, 511-519. [CrossRef] [PubMed]

47. Goletzke, J.; Buyken, A.E.; Louie, J.C.Y.; Moses, R.G.; Brand-Miller, J.C. Dietary micronutrient intake during pregnancy is a function of carbohydrate quality. Am. J. Clin. Nutr. 2015, 102, 626-632. [CrossRef] [PubMed]

48. Kirkpatrick, C.F.; Bolick, J.P.; Kris-Etherton, P.M.; Sikand, G.; Aspry, K.E.; Soffer, D.E.; Willard, K.E.; Maki, K.C. Review of current evidence and clinical recommendations on the effects of low-carbohydrate and very-low-carbohydrate (including ketogenic) diets for the management of body weight and other cardiometabolic risk factors: A scientific statement from the National Lipid Association Nutrition and Lifestyle Task Force. J. Clin. Lipidol. 2019, 13, 689.e1-711.e1.

49. Paterson, P.; Sheath, J.; Taft, P.; Wood, C. Maternal and foetal ketone concentrations in plasma and urine. Lancet 1967, 1, 862-865. [CrossRef]

50. Knopp, R.H.; Magee, M.S.; Raisys, V.; Benedetti, T.; Bonet, B. Hypocaloric diets and ketogenesis in the management of obese gestational diabetic women. J. Am. Coll. Nutr. 1991, 10, 649-667. [CrossRef] [PubMed]

51. Christian, P.; Stewart, C.P. Maternal micronutrient deficiency, fetal development, and the risk of chronic disease. J. Nutr. 2010, 140, 437-445. [CrossRef]

52. Nordic Council of Ministers. Nordic Nutrition Recommendations 2012: Integrating Nutrition and Physical Activity; Nord; Nordic Council of Ministers: Copenhagen, Denmark, 2014. [CrossRef]

53. Wang, J.; Moore, D.; Subramanian, A.; Cheng, K.K.; Toulis, K.A.; Qiu, X.; Saravanan, P.; Price, M.J.; Nirantharakumar, K. Gestational dyslipidaemia and adverse birthweight outcomes: A systematic review and meta-analysis. Obes. Rev. 2018, 19, 1256-1268. [CrossRef]

54. Barbour, L.A.; Farabi, S.S.; Friedman, J.E.; Hirsch, M.N.M.; Reece, M.S.; Van Pelt, R.E.; Hernandez, T.L. Postprandial triglycerides predict newborn fat more strongly than glucose in women with obesity in early pregnancy. Obesity 2018, 26, 1347-1356. [CrossRef]

55. Desoye, G.; Herrera, E. Adipose tissue development and lipid metabolism in the human fetus: The 2020 perspective focusing on maternal diabetes and obesity. Prog. Lipid Res. 2021, 81, 101082. [CrossRef] [PubMed]

56. Barber, T.; Hanson, P.; Kabisch, S.; Pfeiffer, A.; Weickert, M. The low-carbohydrate diet: Short-term metabolic efficacy versus longer-term limitations. Nutrients 2021, 13, 1187. [CrossRef]

57. Barbour, L.A.; Hernandez, T.L. Maternal lipids and fetal overgrowth: Making fat from fat. Clin. Ther. 2018, 40, 1638-1647. [CrossRef]

58. Contreras-Duarte, S.; Carvajal, L.; Garchitorena, M.J.; Subiabre, M.; Fuenzalida, B.; Cantin, C.; Farías, M.; Leiva, A. Gestational diabetes mellitus treatment schemes modify maternal plasma cholesterol levels dependent to women's weight: Possible impact on feto-placental vascular function. Nutrients 2020, 12, 506. [CrossRef]

59. Friedman, J.E. Developmental programming of obesity and diabetes in mouse, monkey, and man in 2018: Where are we headed? Diabetes 2018, 67, 2137-2151. [CrossRef] [PubMed]

60. Brumbaugh, D.E.; Friedman, J.E. Developmental origins of nonalcoholic fatty liver disease. Pediatr. Res. 2014, 75, 140-147. [CrossRef] [PubMed]

61. Hernandez, T.L.; Farabi, S.S.; Hirsch, N.; Dunn, E.Z.; Haugen, E.A.; Brumbaugh, D.; Brown, M.S.; Friedman, J.E.; Barbour, L.A. 352-OR: Maternal triglycerides in gestational diabetes are strongly associated with increased newborn hepatic fat independent of subcutaneous fat. Diabetes 2019, 68 (Suppl. 1), 352. [CrossRef]

62. Westman, E.C.; Feinman, R.D.; Mavropoulos, J.C.; Vernon, M.C.; Volek, J.S.; Wortman, J.A.; Yancy, W.S.; Phinney, S.D. Lowcarbohydrate nutrition and metabolism. Am. J. Clin. Nutr. 2007, 86, 276-284. [CrossRef]

63. Bell, J.D.; Margen, S.; Calloway, D.H. Ketosis, weight loss, uric acid, and nitrogen balance in obese women fed single nutrients at low caloric levels. Metabolism 1969, 18, 193-208. [CrossRef] 